\title{
COMPARISON OF MICROSCOPIC MECHANISMS OF BLUE ANTI-STOKES LUMINESCENCE IN UNDOPED AND CHROMIUM DOPED ZnSe CRYSTALS
}

\author{
V.YU. IVANOV ${ }^{a, *}$, A. OMEL'ChUK ${ }^{b}$ AND M. GODLEWSKI ${ }^{a}$ \\ a Institute of Physics, Polish Academy of Sciences \\ Al. Lotników 32/46, 02-668 Warsaw, Poland \\ ${ }^{b}$ Department of Physics, University of Dnepropetrovsk \\ 320625, Dnepropetrovsk, Ukraine
}

\begin{abstract}
Anti-Stokes luminescence is observed in chromium doped and in undoped ZnSe crystals. In the former case anti-Stokes luminescence is due to two complementary ionization transitions of $\mathrm{Cr}$ ions. In the latter case two-photon absorption becomes important at a high excitation density.
\end{abstract}

PACS numbers: 71.55.Gs, 78.55.Et, 76.30.Fc

Recently we have reported the observation of a strong blue photoluminescence (PL) of ZnSe bulk crystals excited via chromium ionization transitions $[1,2]$. The blue PL, with a zero phonon line at $2.692 \mathrm{eV}$, identified as shallow donor shallow acceptor pair (DAP) PL, was studied. This anti-Stokes luminescence (ASL) dominates the PL spectrum in $\mathrm{ZnSe}: \mathrm{Cr}$ at the excitation energy of $2.41 \mathrm{eV}$ and temperature $T<20 \mathrm{~K}$. The quantum efficiency of the ASL excitation was relatively high and was of about $10^{-3}$. In addition to a simple two-step excitation, due to complementary ionization transitions of chromium ions, acceptor related excitation processes were suggested to be active but expected to be less efficient. In this work we verify this statement by comparing the PL spectra and the ASL efficiency for either chromium doped or undoped ZnSe bulk crystals. We show that the excitation mechanism of the ASL PL observed in undoped ZnSe crystals differs from that in $\mathrm{ZnSe}: \mathrm{Cr}$.

ZnSe crystals studied were grown by either the Bridgman-Stockbarger method (purified by a zone melting with a following treatment in a zinc melt) or by chemical vapor deposition (CVD). The first samples were intentionally doped with chromium, which was introduced to the melt. Chromium concentration, determined from the mass spectral analysis, varied between $10^{16}$ and $5 \times 10^{19} \mathrm{~cm}^{-3}$. In this work we will show the results taken for the sample with a moderate doping

*fax: (48-22) 8430926, e-mail: ivanov@ifpan.edu.pl 
level of $2 \times 10^{18} \mathrm{~cm}^{-3}$. The concentration of unintentional impurities, such as $\mathrm{Cu}$, $\mathrm{Mn}, \mathrm{Fe}, \mathrm{Cl}, \mathrm{Na}, \mathrm{Li}$ was below $10^{16} \mathrm{~cm}^{-3}$. CVD samples were not intentionally doped and had a low contamination level with transition metal ions. We have not observed chromium-related signals in neither electron spin resonance (ESR) nor in PL studies.

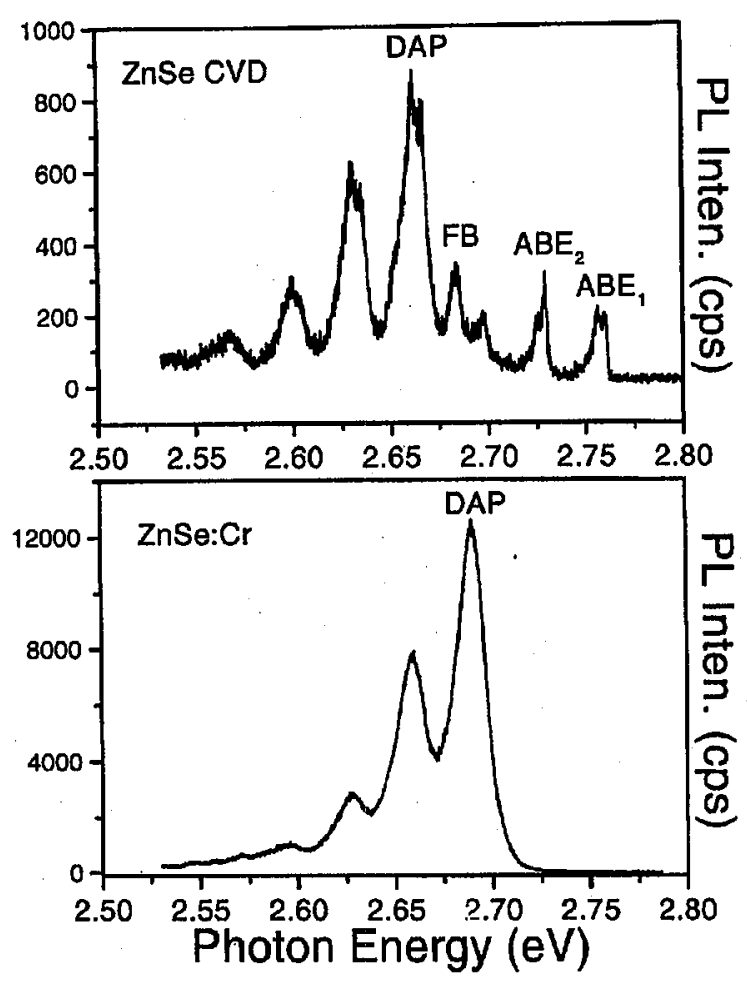

Fig. 1. 2 K ASL spectra observed for Cr-doped and undoped ZnSe samples at the same excitation density. The identity of the $P L$ emissions is shown. $A B E_{1}$ and $A B E_{2}$ stand for two unidentified acceptor bound exciton ( $A B E)$ transitions.

In Fig. 1 we show PL spectra observed for two types of ZnSe crystals studied. Under the ASL excitation conditions (the excitation energy is smaller than the emission energy) PL of $\mathrm{ZnSe}: \mathrm{Cr}$ consists of shallow DAP transition followed by LO-phonon replica. Neither excitonic nor free-to-bound (FB) emissions are observed. In contrast, in the case of CVD samples excitonic transitions are also seen under such excitation conditions. DAP PL in the CVD samples is shifted to slightly lower energy, which is due to differences in contamination of the sample. Its DAP origin was verified from time-resolved PL investigations, which show a typical energy dependence of the PL decay time, which relates to the spatial distribution of the pair distances. However, the chemical origin of the shallow donor and acceptor species active in the DAP transitions remains unknown. 
The present results clearly indicate that a two-step excitation of the ASL is also active in undoped $\mathrm{ZnSe}$ samples. For the ASL observation free electrons and holes should be photo-generated. In undoped CVD samples we explain this by two complementary ionization transitions of deep acceptor responsible for the red PL. The red PL of ZnSe is observed under the ASL excitation conditions and its increase follows the increase in the ASL emission. This two-step process is however by far less efficient than that in chromium doped samples, where it was related to complementary ionization transitions of chronisum ions [1,2]. The spectra shown in Fig. 1, taken at the same excitation density, show that the ASL efficiency in the CVD sample is by 1.5 order in magnitude smaller than that in chromium doped sample.

For the band-to-band excitation DAP PLs are accompanied by FB, acceptor bound $(\mathrm{ABE})$ and donor bound (DBE) exciton transitions. These PL transitions were not seen once free carriers were photo-generated separately in two photoionization transitions. This result suggests that simultaneous excitation of free electrons and holes is necessary for the formation of free and bound excitons. Once free carriers are excited in separate transitions (two-step excitation via $\mathrm{Cr}$ (in $\mathrm{ZnSe}: \mathrm{Cr}$ ) or acceptor (ZnSe CVD) levels) they are trapped by donor and acceptor centers before they can form excitons.

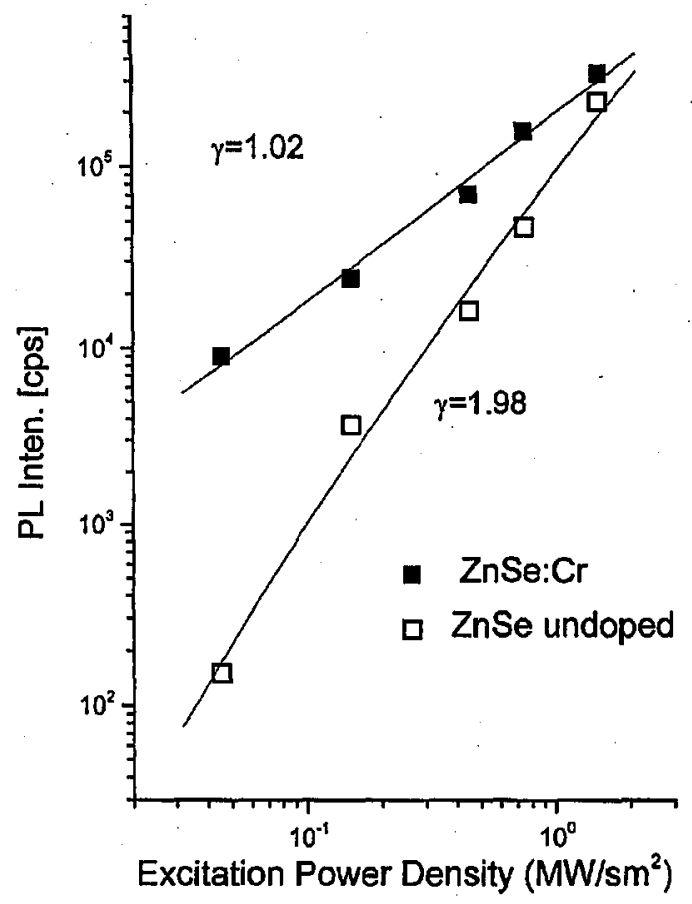

Fig. 2. Dependence of the ASL intensity (DAP transition) on the excitation density in $\mathrm{ZnSe}: \mathrm{Cr}$ and in undoped CVD ZnSe samples. 
The relevant question is why excitonic emissions appear in the ASL of the CVD sample. To explain this observation we have studied ASL in these samples under varying excitation conditions. The excitonic emissions are not observed at low excitation densities. Then, at an increased excitation density they appear and rise fast. Figure 2 shows that a quadratic dependence is observed of the ASL intensity on the excitation density for the CVD sample, but linear for the $\mathrm{Cr}$ doped samples.

Linear dependence of the ASL intensity was explained by metastability of the $\mathrm{Cr}^{+}$excited state of chromium $[1,2]$. In that case two complementary ionization transitions of $\mathrm{Cr}$ ions result in a linear increase in the ASL intensity. For the two-photon absorption process a quadratic dependence should be observed. Such dependence is found for ZnSe CVD sample (see Fig. 2). Thus, the two-photon absorption process apparently becomes dominant under high excitation densities in CVD samples. In contrary, we have not observed evidence for the two-photon absorption process in chromium doped samples. This likely is due to large absorption rates for two ionization transitions of chromium ions observed in absorption and in ESR spectra of $\mathrm{ZnSe}: \mathrm{Cr}$ samples.

Concluding, we show that chromium ions in ZnSe lattice, introducing intense absorption bands for ionization transitions, result in efficient light up-conversion processes. Efficiency of the ASL process in $\mathrm{ZnSe}: \mathrm{Cr}$ is relatively large and is by 1.5 order in magnitude larger than that related to two-photon absorption transitions in undoped ZnSe CVD samples.

This work was partly supported by grant number 2 P03B 01813 of the Committee for Scientific Research (Poland).

\section{References}

[1] V.Yu. Ivanov, Yu.G. Semenov, M. Surma, M. Godlewski, Acta Phys. Pol. A 88, 743 (1995).

[2] V.Yu. Ivanov, Yu.G. Semenov, M. Surma, M. Godlewski, Phys. Rev. B 54, 4696 (1996). 